\title{
МОДЕЛИРОВАНИЕ РАССЕЯНИЯ ЭЛЕКТРОМАГНИТНЫХ ВОЛН НА УГЛОВЫХ СТРУКТУРАХ
}

\section{Преображенский А.П., Чопоров О.Н.}

В работе рассматриваются особенности рассеяния электромагнитных волн на угловых дифракционных структурах. Решение задачи проводится на основе метода интегральных уравнений. Проведен сравнительный анализ характеристик рассеяния структур с различной формой.

Ключевые слова: дифракция; интегральное уравнение; рассеяние радиоволн; радиосвязь; угловая структура; метод моментов; радиоизлучение.

\section{THE SIMULATION \\ OF SCATTERING OF ELECTROMAGNETIC WAVES ON ANGULAR STRUCTURES}

\section{Preobrazhensky A.P., Choporov O.N.}

The paper discusses the characteristics of scattering of electromagnetic waves on the angular diffraction structures. The solution of the problem is based on the method of integral equations. A comparative analysis of the scattering characteristics of structures with different shape is carried out.

Keywords: diffraction; integral equation; scattering of radio waves; telecommunication; angular structure; method of moments; radioirradiation. 
Для современного этапа развития общества характерно то, что повсеместно внедряются инфокоммуникационные комплексы в различные области человеческой деятельности [1-4]. Большой рост количества радиоэлектронных средств определяет высокую территориальную плотность размещения источников радиоизлучения, происходит усложнение электромагнитной обстановки, ухудшается электромагнитная экология [5-8]. При освоении новых частотных диапазонов можно столкнуться с тем, что возникнут технические трудности и финансовые затраты.

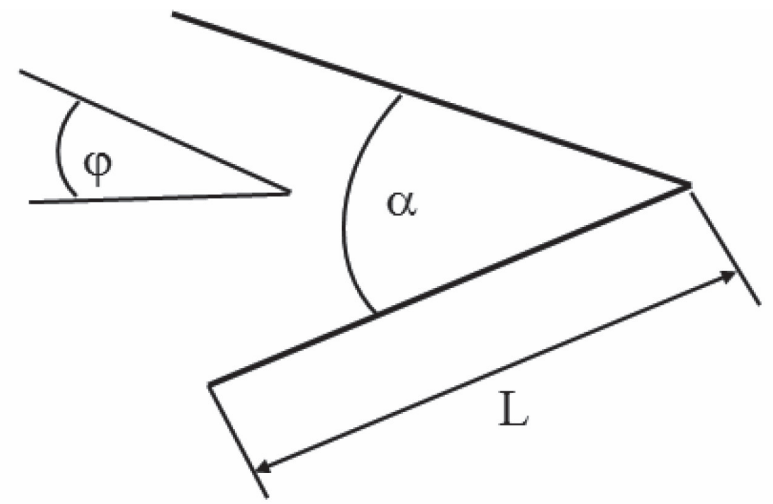

a)

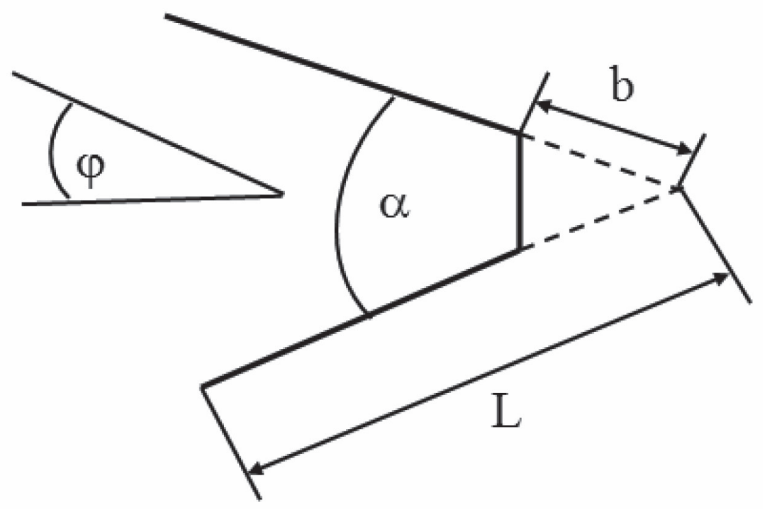

б) 


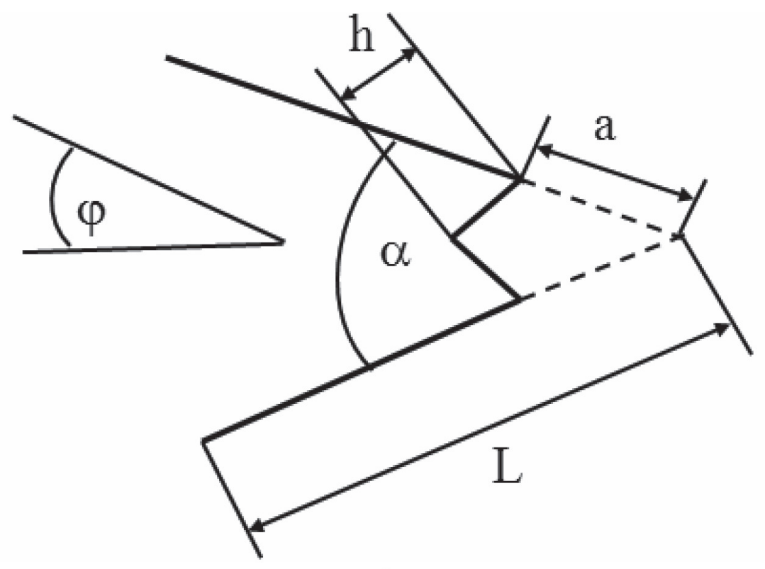

B)

Рис. 1

Среди элементов, входящих в состав радиоэлектронных систем, выделяют большое количество дифракционных структур, формирующих обратное поле рассеяния [9-11]. Представляет интерес рассмотреть особенности рассеяния электромагнитных волн на угловых структурах.

Примеры угловых структур приведены на рис. 1.

Для того, чтобы определить плотности токов на поверхности исследуемой дифракционной структуры, мы воспользуемся уравнением Фредгольма первого рода, в которое входит плотность искомого электрического тока для случая $E$-поляризации [12]:

$$
\frac{\omega \cdot \mu}{4} \cdot \int_{\alpha}^{\beta} j(t) \cdot H_{0}^{2}\left[k \cdot L_{0}(\tau, t)\right] \cdot \sqrt{\xi^{\prime 2}(t)+\eta^{\prime 2}(t)} d t=E_{z}^{0}(\tau),
$$

здесь $L_{0}(\tau, t)=\sqrt{[\xi(\tau)-\xi(t)]^{2}+[\eta(\tau)-\eta(t)]^{2}}-$ представляет собой расстояние между точкой наблюдения и точкой интегрирования, $E_{z}^{0}(\tau)$ - является продольной составляющей для напряженности падающего электромагнитного поля для точки, лежащей на 
контуре. Задание контура структуры осуществляется параметрическим образом: $x=\xi(t), y=\eta(t), \alpha \leq t \leq \beta$, здесь $\xi^{\prime}(t), \eta^{\prime}(t)-$ являются первыми производными соответствующих функций, $k=2 \cdot \pi / \lambda, \lambda$ - обозначает длину падающих электромагнитных волн.

Когда решается интегральное уравнение на основе метода моментов требуется правильным образом описать особенность сингулярности ядра в интегральном уравнении. Например, в случае, когда совпадают координаты точки наблюдения и интегрирования, т. е. при $L_{0} \rightarrow 0$, для двумерной функции Грина мы имеем $H_{0}^{(2)}(k R) \rightarrow \Delta \ell\left[1-\left(\frac{j}{2 \pi}\right)\left[\gamma-1+\ln \left(\frac{k \Delta \ell}{4}\right)\right]\right][13]$, где $\gamma=$ 0,577215664 - обозначает постоянную Эйлера.

На основе полученных в результате решения системы уравнений компонент $\left[J_{m}\right]$ мы определяем рассеянное поле.

3. Базируясь на интеграле Кирхгофа [14] определяем рассеянное электромагнитное поле, которое связано с полученными электрическими токами $J_{z}(r)$ таким образом:

$$
H\left(\theta_{r}\right)=\exp (-j k r) \sqrt{\frac{k}{2 \pi r}} \int_{\ell} J_{z}\left(r^{\prime}\right) \exp \left(j k r^{\prime} \cos \left(\theta_{r}\right)\right) d r^{\prime},
$$

где $\theta_{r}$ - является углом наблюдения; $k$ - волновым числом; $r-$ радиус-вектором точки наблюдения, относящейся к дальней зоне.

4. Эффективная площадь рассеяния (ЭПР) связана с рассеянным электромагнитным полем на основе формулы [15]:

$$
\sigma_{\text {двум }}=2 \pi r\left|H\left(\theta_{r}\right)\right|^{2} \text {. }
$$

Подход, связанный с анализом характеристик рассеяния двумерных структур может быть расширен для того, чтобы осуществлять оценку характеристик рассеяния трехмерных структур [16-18]. На основе приближенной формулы двумерную ЭПР пересчитывают в трехмерную ЭПР [13]:

$$
\sigma_{\text {трех }}=\frac{2 \mathrm{w}^{2}}{\lambda} \sigma_{\text {двум }}
$$

где $\mathrm{w}$ - является размером структуры в направлении, перпендикулярном плоскости чертежа (рис. 1). 


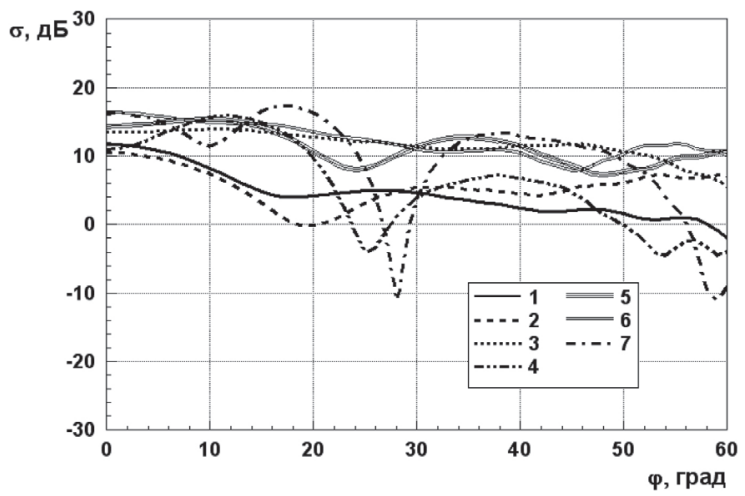

a)

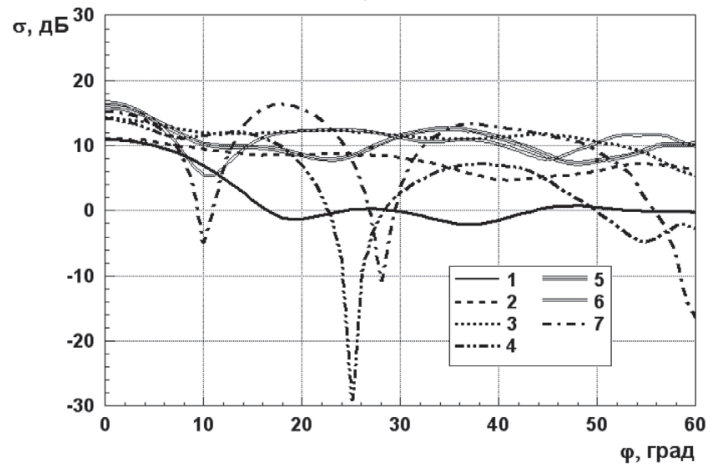

б)

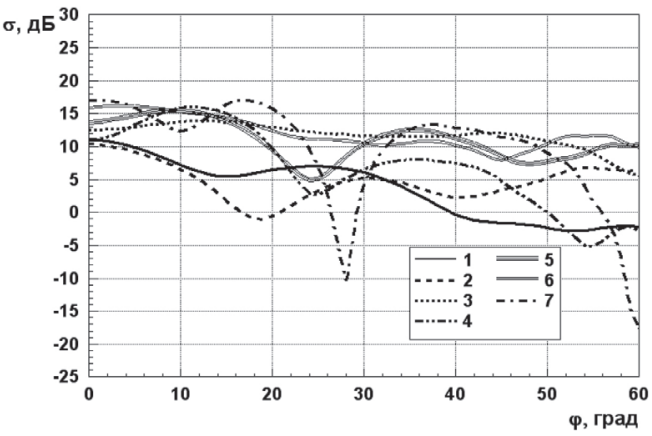

в)

Puc. 2 
На рис. 2 приведены рассчитанные значения ЭПР в зависимости от угла наблюдения. Рис. 2а соответствует рис. 1а, рис. 2б соответствует рис. 16 , рис. 2в соответствует рис. 1в. Кривая 1 соответствует $\mathrm{L}=4 \lambda$, кривая 2 соответствует $\mathrm{L}=5 \lambda$, кривая 3 соответствует $\mathrm{L}=6 \lambda$, кривая 4 соответствует $\mathrm{L}=7 \lambda$, кривая 5 соответствует $\mathrm{L}=8 \lambda$, кривая 6 соответствует $\mathrm{L}=9 \lambda$, кривая 7 соответствует $\mathrm{L}=10 \lambda$. При расчетах $\mathrm{b}=2 \lambda, \mathrm{h}=2 \lambda, \mathrm{a}=20^{\circ}$.

В результате анализа было установлено, что в секторе углов наблюдения от $0^{\circ}$ до $30^{\circ}$ наибольший разброс для ЭПР соответствует случаю, приведенному на рис 16 . При изменении L от $4 \lambda$ до $10 \lambda$ величина средней ЭПР для сектора углов наблюдения от $5^{\circ}$ до $90^{\circ}$ изменялась не более, чем на 0.3 дБ.

\section{Вывод}

В работе на основе метода интегральных уравнений проведено моделирование и исследованы характеристики рассеяния угловых дифракционных структур.

\section{Список литературы}

1. Баранов А.В. Проблемы функционирования mesh-сетей // Вестник Воронежского института высоких технологий. 2012. № 9. C. 49-50.

2. Милошенко О.В. Методы оценки характеристик распространения радиоволн в системах подвижной радиосвязи // Вестник Воронежского института высоких технологий. 2012. № 9. C. 60-62.

3. Мишин Я.А. О системах автоматизированного проектирования в беспроводных сетях // Вестник Воронежского института высоких технологий. 2013. № 10. С. 153-156.

4. Головинов С.О., Хромых А.А. Проблемы управления системами мобильной связи // Вестник Воронежского института высоких технологий. 2012. № 9. С. 13-14. 
5. Кульнева Е.Ю., Гащенко И.А. О характеристиках, влияющих на моделирование радиотехнических устройств // Современные наукоемкие технологии. 2014. № 5-2. С. 50.

6. Казаков Е.Н. Разработка и программная реализации алгоритма оценки уровня сигнала в сети wi-fi // Моделирование, оптимизация и информационные технологии. 2016. № 1. С. 13.

7. Канавин С. В., Лукьянов А. С. Перспективы применения систем мобильного широкополосного доступа в сетях подвижной радиосвязи на основе стандартов mobile WIMAX и LTE // Вестник Воронежского института высоких технологий. 2016. № 16. C. $79-82$.

8. Глотова Т.В. Применение гибридного метода для расчета характеристик рассеяния объектов над шероховатой поверхностью // Моделирование, оптимизация и информационные технологии. 2016. № 1. С. 11.

9. Болучевская О.А., Горбенко О.Н. Свойства методов оценки характеристик рассеяния электромагнитных волн // Моделирование, оптимизация и информационные технологии. 2013. № 3. С. 4.

10. Максимова А.А., Юрочкин А.Г. Методы исследования характеристик рассеяния электромагнитных волн объектами // Вестник Воронежского института высоких технологий. 2016. № 16. C. 53-56.

11. Пекшев Г.А., Скляр А.Г. Некоторые свойства лучевых методов, используемых для анализа распространения электромагнитных волн // В мире научных открытий. 2015. № 12. С. 17-23.

12.Захаров Е.В., Пименов Ю.В. Численные методы решения задач дифракции. М.: Радио и связь, 1986. 184 с.

13. Ling H. RCS of waveguide cavities: a hybrid boundary-integral/modal approach. // IEEE Trans. Antennas Propagat., 1990, vol. AP-38, no. 9, pp. 1413-1420.

14.Стрэттон Дж. Теория электромагнетизма. М.Л.: Гостехиздат, 1948. 539 c. 
15. Справочник по радиолокации / Под ред. Сколника М. М.: Сов. радио, 1976. т.1. 455 с.

16. Ерасов С.В. Оптимизационные процессы в электродинамических задачах // Вестник Воронежского института высоких технологий. 2013. № 10. С. 20-26.

17. Щербатых C.С. Метод интегральных уравнений как основной способ анализа в САПР антенн // Моделирование, оптимизация и информационные технологии. 2016. № 1. С. 10.

18.Пекшев Г.А., Скляр А.Г. Проблемы управления рассеянными электромагнитными полями // В мире научных открытий. 2015. № 12. C. 24-30.

\section{References}

1. Baranov A.V. Vestnik Voronezhskogo instituta vysokikh tekhnologiy. 2012. № 9, pp. 49-50.

2. Miloshenko O.V. Vestnik Voronezhskogo instituta vysokikh tekhnologiy. 2012. № 9, pp. 60-62.

3. Mishin Ya.A. Vestnik Voronezhskogo instituta vysokikh tekhnologiy. 2013. № 10, pp. 153-156.

4. Golovinov S.O., Khromykh A.A. Vestnik Voronezhskogo instituta vysokikh tekhnologiy. 2012. № 9, pp. 13-14.

5. Kul'neva E.Yu., Gashchenko I.A. Sovremennye naukoemkie tekhnologii. 2014. № 5-2. P. 50.

6. Kazakov E.N. Modelirovanie, optimizatsiya i informatsionnye tekhnologii. 2016. № 1. P. 13.

7. Kanavin S.V., Luk'yanov A.S. Vestnik Voronezhskogo instituta vysokikh tekhnologiy. 2016. № 16, pp. 79-82.

8. Glotova T.V. Modelirovanie, optimizatsiya i informatsionnye tekhnologii. 2016. № 1. P. 11.

9. Boluchevskaya O.A., Gorbenko O.N. Modelirovanie, optimizatsiya $i$ informatsionnye tekhnologii. 2013. № 3. P. 4.

10. Maksimova A.A., Yurochkin A.G. Vestnik Voronezhskogo instituta vysokikh tekhnologiy. 2016. № 16, pp. 53-56. 
11. Pekshev G.A., Sklyar A.G. V mire nauchnykh otkrytiy. 2015. № 12, pp. 17-23.

12.Zakharov E.V., Pimenov Yu.V. Chislennye metody resheniya zadach difraktsii [Numerical methods for solving diffraction problems]. M.: Radio i svyaz', 1986. 184 p.

13. Ling H. RCS of waveguide cavities: a hybrid boundary-integral/modal approach. IEEE Trans. Antennas Propagat., 1990, vol. AP-38, no. 9, pp. 1413-1420.

14. Stretton Dzh. Teoriya elektromagnetizma [The theory of electromagnetism]. M.L.: Gostekhizdat, 1948. 539 p.

15.Spravochnik po radiolokatsii [Reference radar] / Skolnik M. (ed.). Sov. radio, 1976. V.1. 455 p.

16.Erasov S.V. Vestnik Voronezhskogo instituta vysokikh tekhnologiy. 2013. № 10, pp. 20-26.

17. Shcherbatykh S.S. Modelirovanie, optimizatsiya i informatsionnye tekhnologii. 2016. № 1. P. 10.

18.Pekshev G.A., Sklyar A.G. V mire nauchnykh otkrytiy. 2015. № 12, pp. 24-30.

\section{ДАННЫЕ ОБ АВТОРАХ}

Преображенский А.П., Чопоров О.Н.

Воронежский институт высоких технологий

ул. Ленина, 73А, Воронеж, Воронежская обл., 394043, Российская Федерация

komkovvivt@ya.ru

\section{DATA ABOUT THE AUTHORS}

\section{Preobrazhensky A.P., Choporov O.N.}

Voronezh Institute of High Technologies

73A, Lenin Str., Voronezh region, 394043, Russian Federation

komkovvivt@ya.ru 